\title{
Towards efficient and targeted delivery of nutrient and micronutrient nanomaterials in crop plants
}

\author{
GREGORY V. LOWRY ${ }^{1}$, YILIN ZHANG ${ }^{1}$, JUAN PABLO \\ GIRALDO $^{2}$ AND ROBERT D. TILTON ${ }^{1}$ \\ ${ }^{1}$ Carnegie Mellon University \\ ${ }^{2}$ University of California, Riverside \\ Presenting Author: glowry@cmu.edu
}

Sustainable agriculture will require highly efficient delivery of nutrients and micronutrients, and the ability to protect crops against climate change induced stress. Nano-enabled drug delivery approaches developed for humans could be leveraged for efficient and targeted delivery of micronutrient nanoparticles in plants, but the "rules" controlling nanomaterial-plant interactions have not yet been elucidated. Moreover, nanoenabled carriers that can respond to the plant's biological needs or to environmental conditions (e.g. temperature) to deliver nanoparticulate micronutrients to desired locations in plants are not yet available. Here, we used star polymer nanoparticles with precisely controlled sizes ( $6 \mathrm{~nm}$ to $35 \mathrm{~nm}$ ), negative charge content (17-83\% PAA content) and hydrophobicity (different MSEA/MTEA ratios) to systematically evaluate how nanoparticle size, charge, and hydrophobicity affect their uptake, phloem loading, and distribution in tomato (Solanum lycopersicum) plants. These foliar applied nanoparticles had rapid and high rates of phloem loading $(\sim 30 \mathrm{wt} \%)$. The property differences affected their distribution to other plant compartments including younger leaves, older leaves, stems and roots. The smallest $\sim 6 \mathrm{~nm}$ star polymers translocate most to younger leaves, while the largest $\sim 35 \mathrm{~nm}$ star polymer moved preferentially to roots, likely due to different transport pathways in the leaf mesophyll tissue for the different sized materials. The lower charge content star polymer moved more into the nonvascular tissues (younger leaf, older leaf and root), while the higher charge content star polymers mainly stayed in the stem. This is likely due to charge dependent interactions with cell walls. Hydrophobicity did not affect star polymer translocation after entering the mesophyll but did affect their interaction with the leaf surface and the ability to penetrate the epidermis. Variations of these star polymers were also synthesized, and were able to provide temperature programed or ROS instigated in vivo release of nutrients. These results can promote rational design of metal and metal oxide NPs for more efficient and targeted agent delivery in plants, and can provide a platform for delivering nutrients and micronutrients in a plant-responsive and environmentally responsive manner to combat climate-related stress and improve agricultural sustainability. 